\title{
Clinical needs and technical requirements for ventilators for COVID-19 treatment critical patients: an evidence-based comparison for adult and pediatric age
}

\author{
Martina Andellini ${ }^{1,2}$ (D) $\cdot$ Simone De Santis ${ }^{3} \cdot$ Federico Nocchi $^{4} \cdot$ Elena Bassanelli ${ }^{3} \cdot$ Leandro Pecchia $^{2} \cdot$ Matteo Ritrovato $^{1,3}$
}

Received: 8 June 2020 / Accepted: 24 July 2020 / Published online: 30 July 2020

(C) IUPESM and Springer-Verlag GmbH Germany, part of Springer Nature 2020, corrected publication 2020

\begin{abstract}
The spread of severe acute respiratory syndrome coronavirus 2, taking on pandemic proportions, is placing extraordinary and unprecedented demands on healthcare systems worldwide. The increasing number of critical patients who, experiencing respiratory failure from acute respiratory distress syndrome, need respiratory support, has been leading countries to race against time in arranging new Intensive Care Units (ICUs) and in finding affordable and practical solutions to manage patients in each stage of the disease. The simultaneous worldwide emergency caused serious problems for mechanical ventilators supply. This chaotic scenario generated, indeed, a frenetic race to buy life-saving ventilators. However, the variety of mechanical ventilators designs, together with the limitations in time and resources, make the decision-making processes on ventilators procurement crucial and not counterbalanced by the evaluation of devices quality. This paper aimed at offering an overview of how evidence-based approach for health technologies evaluation, might provide support during Corona Virus Disease 2019 (COVID-19) pandemic in ICUs management and critical equipment supply. We compared and combined all the publicly available indications on the essential requirements that ICU ventilators might meet to be considered acceptable for treating COVID-19 patients in severe to critical illnesses. We hope that the critical analysis of these data might help readers to understand how structured decision-making processes based on evidence, evaluating the safety and effectiveness of a given medical device and the effects of its introduction in a healthcare setting, are able to optimize time and resources allocation that should be considered essential, especially during pandemic period.
\end{abstract}

Keywords Intensive care ventilators · Mechanical ventilators · COVID-19 · Emergency · Ventilators' essential technical requirements $\cdot$ Pediatric

This article is part of the COVID-19 Health Technology: Design, Regulation, Management, Assessment

Martina Andellini

martina.andellini@opbg.net

1 HTA Unit, Bambino Gesù Children's Hospital, Viale di Villa Pamphili 100, 00152 Rome, Italy

2 School of Engineering, University of Warwick, Coventry CV4 7AL, UK

3 HTA \& Safety Research Unit, Bambino Gesù Children's Hospital, Rome, Italy

4 Clinical Engineering Department, Bambino Gesù Children's Hospital, Rome, Italy

\section{Introduction}

The Severe Acute Respiratory Syndrome - Coronavirus ([SARS]-CoV-2) was identified in December 2019 from a group of patients presenting severe pneumonia symptoms in Wuhan, China [1, 2]. From that moment to April 22, 2020, the Corona Virus Disease 2019 (COVID-19) caused more than 7,016,794 confirmed cases and more than 402,874 deaths worldwide. ${ }^{1}$ A report from the Chinese Centre for Disease Control and Prevention [3], including approximately 44,500 COVID-19 confirmed cases, reported that $81 \%$ of patients showed mild symptoms, $14 \%$ severe disease and 5\% critical illnesses with an overall case fatality rate of $2.3 \%$. Data from the same report showed that $87 \%$ of patients were between 30 and 79 years old, $3 \%$ were

\footnotetext{
$\overline{1}$ https://gisanddata.maps.arcgis.com/apps/opsdashboard/index.html\#/ bda7594740fd40299423467b48e9ecf6
} 
80 years old or older, while relatively few cases of infants confirmed with COVID-19 have been reported (1\% were aged 9 years or younger, $1 \%$ were between 10 to 19 years old), and they experienced principally mild illness. It appears as a respiratory infection that can vary from mild respiratory symptoms with spontaneous resolution, to severe pneumonia that in some cases, can be fatal. Data showed that patients who experienced severe clinical symptoms revealed diffuse alveolar damage resulting in end-stage respiratory failure. Unfortunately, at present time even if several therapies have proven to be effective, none of them has been a game changer so far. Severe cases, presenting acute respiratory failure, can receive only respiratory support therapies throughout ventilators (invasive and non-invasive) [4].

Currently available data, indeed, suggest that a significant number of subjects diagnosed with infection from COVID-19 presented acute respiratory failure demanding for Intensive Care Unit (ICU) admissions.

However, data collection methods significantly vary across countries, reflecting the inhomogeneity in representing the ratio between the number of patients who required intensive care and the total number of those hospitalized.

In China, data updated to February 11th, 2020, showed that slightly less than $20 \%$ of the infected population required hospitalization, comprising $14 \%$ of cases with severe symptoms and about $5 \%$ of critical cases that required intensive care [3]. In Italy, from February 24th, 2020 to today, the ratio between patients who required intensive care and the total of those hospitalized halved $^{2}$ (from $20 \%$ to 10\%). Data from France suggested that this ratio even today is $18 \% .^{3}$

In addition to the significant number of COVID-19 patients who have been requiring intensive care treatment, data of patients' permanence in ICUs who required ventilation support significantly vary from few days to several weeks.

Data from China, for instance, reports that patients' require a mean of 12.8 days of respiratory support [5]. In Italy, where the mean age of patients is much higher, data show that some patients need ventilation for several weeks [6]. These differences in duration and intensity of ICU treatments for COVID19 patients depend on several factors including age and comorbidities together with the total number of patients in the country, which could affect the time delay to receive care, ICUs capacities and the availability of COVID-19 rapid testing.

Compared to the duration of stay in ICU for patients with community-acquired pneumonia in influenza season, these values appear extremely higher and vary widely across countries. A paper published in 2018 showed that in Turkey, during a non-emergency situation, the median duration of stay in ICU for Patients with Community-Acquired Pneumonia in influenza season was 5.0 days [7]. It is a general knowledge that hospital admissions due to pneumonia are likely to

\footnotetext{
$\overline{2}$ http://www.salute.gov.it/portale/home.html

3 https://www.gouvernement.fr/info-coronavirus/carte-et-donnees
}

increase during influenza outbreaks. It is common knowledge that longer duration of ICU stay may lead to problems in terms of beds and hospital resources allocation. This concept is exasperated during this global pandemic, which has been straining the health care systems worldwide.

As reference, let's analyse the Italian scenario. In Italy, before the COVID-19 emergency, the number of ICU beds was 5090 [8]. Assuming all the ICU beds are available for 365 days (i.e., no downtime), this equate to a max capability (i.e., 5090 ICU beds $\times 365$ days) of $1,857,850$ ICU bed-days in 12 months (equates 154,820 per month). The number of COVID-19 admissions in Italian ICUs was 4068 from March 5th to April 4th with an average length of stay of 30 days (compared to the normal ICU stay of 14 days) [9].This equates to a demand of 122,040 ICU bed-days in one month just for COVID-19, which is the $79 \%$ of Italian ICU monthly capability. Considering that on average $48.3 \%$ of ICU beds are occupied for other than COVID-19 patients, this creates a saturation of Italian ICUs, with a demand of extra 41,998 ICU bed-days per month (i.e., 1399.98 novel ICU beds required). It must be remarked that ICU saturation affects all the hospitalizations. In fact, many clinical procedures and elective surgeries cannot be performed if ICU beds are not available, in case of complications. However, the real situation is even worse than the estimation presented above by the fact that the infected people and the consequent demand for ICU beds in March in Italy was not equally distributed on the entire country, but was almost exclusively limited to Northern regions.

As this situation is common to most countries worldwide, based on data acquired until now, World Health Organization (WHO) published its recommendations for the European Region as a technical guidance for health systems to respond to COVID-19 outbreak for increasing ICU surge capacity [10]. In Italy, for instance, similar indications aiming at strengthening ICU departments were published on March 4th, 2020. In order to deal with the unexpected influx of patients, the Italian hospitals drastically increased the number of ICU beds, as well as ICU staff and life-saving ventilators and other related supplies [11]. As a direct consequence, and following the example of China, some countries decided, where possible, to build new hospitals, others to reshape several departments into ICUs, others to create new ICUs into buildings different from hospitals or to renovate old hospitals to become new COVID-19 centres.

However, increasing ICU beds means increasing the related medical equipment including ICU ventilators. These life support devices, providing temporary or permanent respiratory assistance to patients who cannot breathe on their own, or who require assistance maintaining adequate ventilation because of illness [12], were agreed to be considered pivotal to the care of COVID-19 critical patients.

During this critical situation, in which countries are racing against time in arranging new ICUs, the main problem of ventilators' supply has emerged. The high technological 
complexity of these devices makes the time required for their production crucial. In addition, as life support devices, ventilators have to pass robust regulatory tests before they are receiving the approval and can be delivered to hospitals.

Considering the complicated variety of ICU ventilators designs, currently offered by a number of manufacturers, together with the limitations in time and resources during the emergency, stakeholders necessitate affordable solutions to rapidly understand the real needs of a specific health context. This means learning how to correctly evaluate the coherent amount of ventilators really needed in a specific context as well as the essential technical requirements that ventilators should have to ensure effective treatment for COVID-19 critical patients.

On April 4th, for instance, a number of UK journals reported that the more than 250 ICU ventilators purchased from China, as an important step in the country's fight against the COVID-19 outbreak, were ditched because serious concerns over the basic quality of ventilators emerged. UK Government was looking forward to the withdrawal and replacement of these ventilators with devices able to ensure safety and effectiveness in providing ventilation for critical patients $[13,14]$. This reflects that the current pandemic emergency is requiring multidisciplinary efforts to evaluate ICU ventilators costeffectiveness.

We wrote this manuscript aiming at offering an overview of how the application of a structured and reliable evidencebased approach for technologies evaluation might provide support during COVID-19 pandemic, resuming and providing all the main information currently publicly available on the essential requirements for the ventilators' management for critical patients.

The expression "evidence-based" is well known in the medical field, while it has been spreading in biomedical engineering field in the recent years.

As evidence-based medicine is based on using the best available evidence to make decisions about individual patients' care, evidence-based clinical engineering uses the best available evidence to make decisions on medical devices and healthcare setting.

Historically, clinical engineering has been based on best practices and clinical engineers have been less involved in research activities as well as in publishing their activities' results in peer reviewed journals. However, in the past 10 years, clinical engineering has been growing globally [15], with the first peer reviewed journal papers on evidence-based being published in 2019 [16, 17]. Similarly to what Pecchia et al. did for personal protective equipment [18], starting from the clinical indications spread by WHO and the Italian Ministry of Health, we joined our efforts to conscientiously share the minimum technical requirements that an ICU ventilator might meet to be considered acceptable for treating COVID-19 patients in severe to critical illnesses, comparing and, at the same time, combining in an easily readable way, all the indications publicly available in order to provide a unified and global vision.

We hope this could significantly help and support manufacturers in designing a basic product which is able to provide a sufficient ventilation support for COVID-19 patients, and, on the other side, hospitals decision-makers in prioritizing, during the COVID-19 pandemic, the most important mechanical ventilators' technical characteristics for the definition of tender specifications.

\section{Clinical indications for suspected and confirmed COVID-19 patients}

A general search on PubMed and google search engines was carried out in order to retrieve as much information as possible about indications and guidelines for the management of suspected and confirmed COVID-19 patients.

The most relevant documents were gathered from the Italian Ministry of Health ${ }^{4}$ and from $\mathrm{WHO}^{5}$ web pages. The two health organizations provided guidelines to manage severe acute respiratory infections in suspected and confirmed COVID-19 patients. As the online documentation confirmed, they agreed on the suggested strategies to manage these critical patients.

WHO provided a detailed description of the clinical syndromes' profiles associated with COVID-19 for mild, severe and critical illnesses and it suggested strategies to manage patients for each of the described conditions [19].

As the patients who experienced mild symptoms do not need ventilation support, our attention was focused on patients with severe and critical illnesses.

Considering adults with severe symptoms, the two health organizations suggested to start the oxygen therapy at $5 \mathrm{~L} / \mathrm{min}$ and titrate flow rates to reach target $\mathrm{SpO} 2 \geq 90 \%$ in nonpregnant adults and children, $\mathrm{SpO} 2 \geq 92-95 \%$ in pregnant patients [20,21].

They strongly recommended to closely monitor these patients in order to early detect any signs of clinical decline and, if necessary, immediately turn to mechanical ventilation.

For those patients with Acute Respiratory Distress Syndrome (ARDS) who required endotracheal intubation they recommended to use a low Tidal Volume (TV) $(4-8 \mathrm{~mL} / \mathrm{kg}$ per predicted body weight, $\mathrm{PBW}$ ) with a plateau pressure (Pplat) of less than $26-28 \mathrm{cmH}_{2} \mathrm{O}$ and a driving pressure less than $12-14 \mathrm{cmH}_{2} \mathrm{O}[19,22]$. WHO claimed that for children, TV should be included in the range $3-6 \mathrm{~mL} / \mathrm{kg}$ PBW in the case of poor respiratory system compliance, or in the range 5$8 \mathrm{~mL} / \mathrm{kg}$ PBW if respiratory system compliance is better preserved [23].

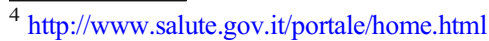

${ }^{5}$ https://www.who.int/
} 
The Italian Ministry of Health also suggested to use higher Positive end-expiratory pressure (PEEP) rather than lower PEEP in patients with moderate and severe ARDS. A table was provided, indicating the possible $\mathrm{PEEP} / \mathrm{FiO}{ }_{2}(\mathrm{FiO} 2$ is the Fraction of inspired oxygen) combinations to obtain the following therapeutic objectives: Oxygen Saturation $\left(\mathrm{SpO}_{2}\right): 88-$ $95 \%, \mathrm{PaO}_{2}: 55-80 \mathrm{mmHg}$, Pplat less than $26 \mathrm{cmH}_{2} \mathrm{O}$, or less than $28 \mathrm{cmH}_{2} \mathrm{O}$ if Body Mass Index (BMI) resulted greater than $30 \mathrm{~kg} / \mathrm{m} 2$, and a driving pressure less than $12 \mathrm{cmH}_{2} \mathrm{O}$. [22]

Moreover, WHO [19] suggested to treat ARDS patients with hypoxemic respiratory failure with non-invasive or high-flow oxygen systems, even if they should be closely monitored because they are likely to clinical decline and, in the case of Non-invasive ventilation (NIV) strategy failure, the patient needs immediate endotracheal intubation.

\section{Essential technical requirements for ICU ventilators}

The urgent demand for ICU ventilators to guarantee continuous care for COVID-19 patients has been leading the major health organizations to publish the minimum acceptable technical requirements for ventilators to be used during the current pandemic.

In this respect, a detailed search of the publicly available indications for mechanical ventilators' essential technical requirements was carried out in order to resume and compare all the indications that the major worldwide health organizations are providing during this time.

In our analysis we considered data from UK National Health Service (NHS) [24, 25], WHO [12] and CONSIP S.p.A. [26] (held by the Italian Ministry of Economy and Finance, it is the purchasing centre of the Italian Public Administration sector).

Table 1 summarizes the three directions. A number of technical specifications were grouped into eleven classes: (i) controls/setting ranges, (ii) invasive and non-invasive ventilation modes, (iii) patient assessment tools, (iv) integrated capabilities, (v) monitored/displayed parameters, (vi) patient alarms, (vii) equipment alarms, (viii) display, (ix) patient transport capability, (x) on-board air compressor or turbine, (xi) internal back-up battery. Each detailed indicator has been made explicit according to the data retrieved from the three organizations websites.

Taking into account the clinical guidelines for suspected and confirmed COVID-19 adult and pediatric patients discussed above, even if the three organizations provided different ranges for ventilators parameters, they do not appear significantly different from each other. More specifically, while CONSIP S.p.A. and WHO provided indication for adult and pediatric patients, the information retrieved from NHS focused only on adults.

The indication for TV, as well as that for plateau airway pressure, are guaranteed by the minimal technical specifications in terms of both volume and pressure. The former clinical requirement is ensured because a TV less than $1000 \mathrm{ml}$ is enough for ventilating a patient with a predicted body weight up to $125-250 \mathrm{~kg}$ (considering the WHO indication of 4-8 ml/ $\mathrm{kg})$. As for the latter, the ranges of Inspiratory Pressure (0-40 $\left.\mathrm{cmH}_{2} \mathrm{O}\right)$ and PEEP $\left(0-20 \mathrm{cmH}_{2} \mathrm{O}\right)$ allow the clinician to adjust for the Pplat and driving pressure values recommended.

Regarding the Respiratory Rate (RR), WHO suggested a range of 10-60 acts per minutes, NHS recommended a range of 10-30 acts per minutes, whereas CONSIP S.p.A did not specify any RR values.

Moreover, the ventilator must accurately control the fraction of inspired oxygen (FiO2) provided to the patient, allowing its full-range adjustment (21-100\% for CONSIP S.p.A and WHO versus $30-100 \%$ for NHS) to meet the different patients' health needs. The ratio of the duration of inspiratory and expiratory phases needs to be regulated (NHS specified the range $1: 1-1: 3$ ) in order to allow the full expiration of the diseased lung, to increase $\mathrm{CO} 2$ clearance and to prevent gas trapping. A feature for air leaks compensation is also required by CONSIP S.p.A, so that the ventilator can adjust the delivered flow accordingly: leaks in the breathing circuit can determine a different volume than the one selected and prevent accurate PEEP supply and gas flow measure.

The ventilator must be provided with several ventilation modes. The three organizations agreed that volumecontrolled (which still guarantees the selected TV when changes in lung compliance and/or in airway resistance occur) and pressure-controlled (particularly for ARDS patients, since they facilitate lung recruitment) ventilation modes should be guaranteed.

For ventilation modes that rely on recognition of patient's efforts, trigger detection systems, both flow-based and pressure-based, are required. More specifically, CONSIP S.p.A required that the device must be provided with Continuous Mandatory Ventilation modes (CMV), Pressure and Volume Assisted/Controlled Ventilation modes (P/VCAC) and for patients partly capable of breathing alone, NHS and WHO suggested that the ventilator should be equipped with Synchronized Intermittent Mandatory Ventilation (SIMV) mode.

In addition, for CONSIP S.p.A and WHO, Pressure Support Ventilation (PSV) mode is required for patients capable of spontaneous breathing: a mode in which every respiratory act is still aided by the device, in order to prevent the risk of barotrauma and to decrease work of breathing.

The capability of switching to NIV, including Continuous Positive Airway Pressure (CPAP) and Bilevel Positive Airway Pressure (BIPAP) modes, is also required by 


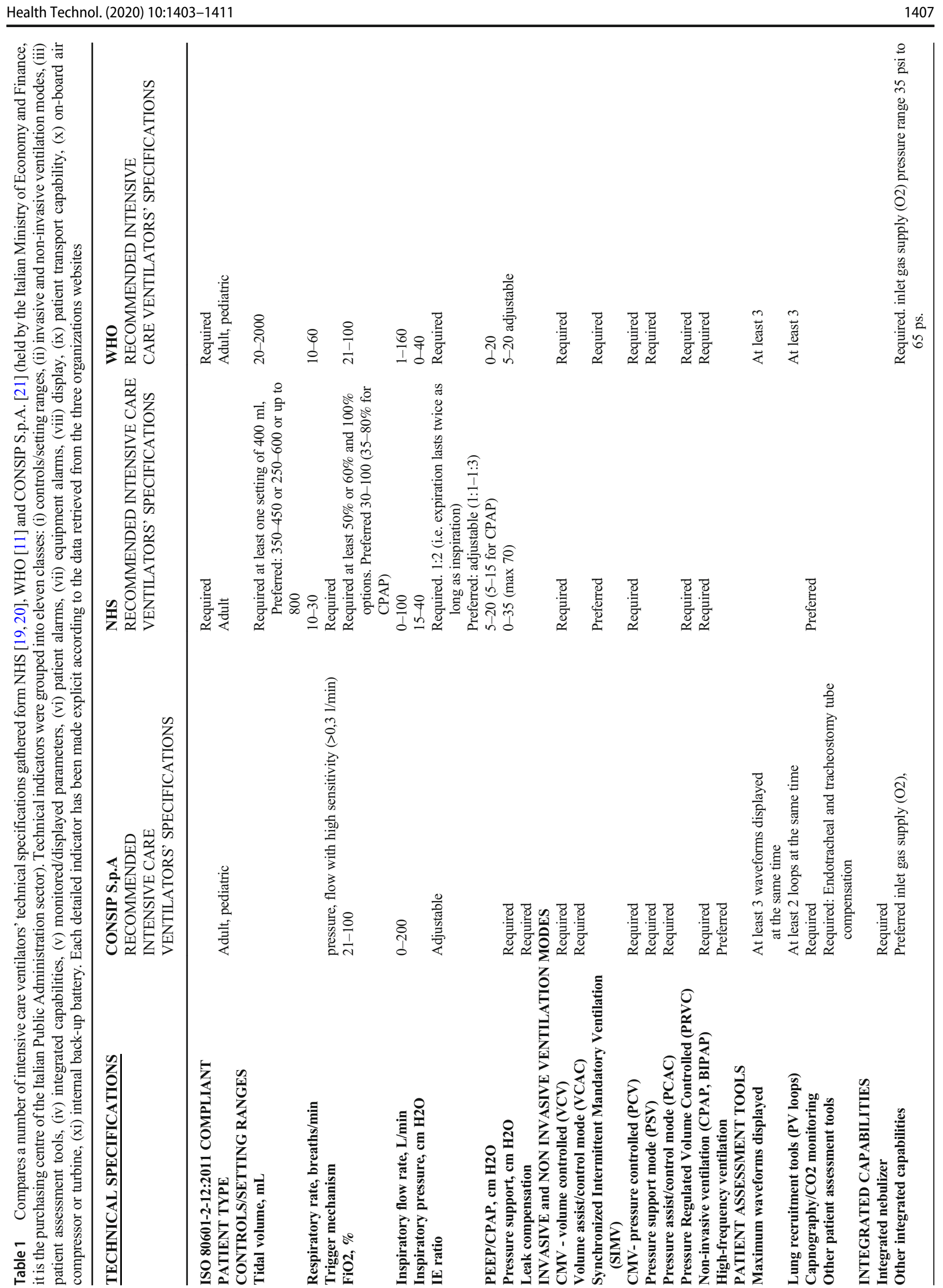




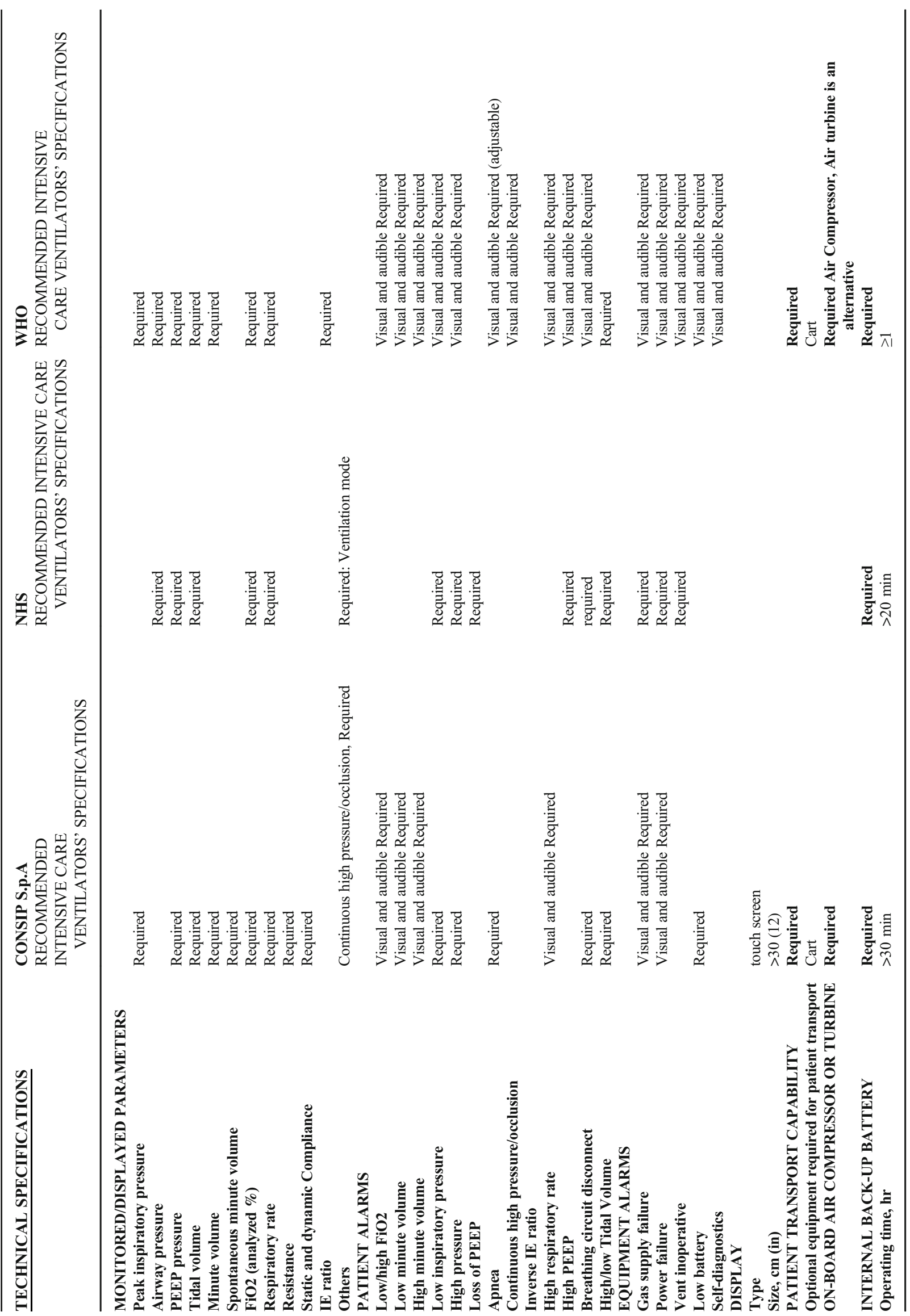


CONSIP S.p.A and WHO for those patients with less need for respiratory support, or during weaning from invasive ventilation.

More advanced ventilation modes, like Pressure Regulated Volume Controlled (PRVC) and High Frequency Oscillatory (HFO) ventilation, are also required. NHS and WHO agreed that PRVC, through which the selected volume is provided at the lowest possible pressure level, is needed. CONSIP S.p.A, instead, recommended the inclusion of HFO mode, considering more technologically complex ventilators, since they need to generate a high frequency ventilation, combined with conventional ventilation modes in some commercially available neonatal ventilators and less frequently used to ventilate adult patients.

Regarding the monitoring of the ongoing treatment, the three organizations agreed that the display of the device should show numeric values for the main respiratory parameters (TV, PEEP, FiO2, respiratory rate). Furthermore, monitoring of both static and dynamic lung compliance (typically lower in ARDS patients) and airway resistance is required by CONSIP S.p.A. More sophisticated tools for the visualization of respiratory mechanics could allow for a better assessment of respiratory function and the obstructive or restrictive nature of disorders. CONSIP S.p.A and WHO believe that the device must also allow showing at least three respiratory parameters' waveforms at the same time, which are important for the visual assessment of ventilation trends and setting optimization. Pressure/volume and flow/volume loops are also required, in order to allow the clinician observe possible pulmonary recruitment anomalies and obstructive or restrictive alterations. A touchscreen and sufficiently large $\left(>12^{\prime \prime}\right)$ display is required, for a quick and easy use by the clinicians.

The integration of a capnography/CO2 monitoring system is agreed by CONSIP S.p.A and NHS for a more accurate assessment of cause and severity of respiratory disorders, and for a guidance to therapeutic choices (and their follow-up).

Furthermore, CONSIP S.p.A suggested the inclusion of a system for endotracheal/tracheostomy tubes' pressure drops compensation for a more accurate ventilation.

The device must be provided with several patient alarms, which must be adequately visible and audible even in the noisy ICU environment. CONSIP S.p.A and WHO agreed that the system must check for high and low values of the main respiratory parameters $(\mathrm{FiO} 2$, minute volume, apnea, respiratory rate, $\mathrm{PEEP}$ ) to alert the clinician of possible changes in the patient's condition. Furthermore, the three organizations agreed for the low and high inspiratory pressure alarm as well as for high PEEP, high/low TV and for the breathing circuit disconnection.

Alarms must also warn about device malfunctions (breathing circuit disconnection, power failure, supply gas failure, low battery). Finally, the device must be provided with an internal battery for backup in case of power supply failure and for in-hospital transport (for which the ventilator must be equipped with a cart).

As discussed, some differences can be found between the minimal specifications recommended by the three organizations taken into account.

NHS's respiratory parameters' ranges (TV, respiratory rate, $\mathrm{FiO}_{2}$, inspiratory flow, inspiratory pressure, Inspiratory:Expiratory ratio (I:E ratio), PEEP, pressure support) are less demanding than WHO's specifications.

NHS also recommends fewer ventilation modes, not requiring PSV nor assist/control modes (VCAC, PCAC), as well as fewer alarms than WHO and CONSIP S.p.A. Moreover, while CONSIP S.p.A resulted more demanding regarding the recommendations for monitored/displayed parameters than NHS and $\mathrm{WHO}$, it is somewhat vague about respiratory parameters; meanwhile, it provides detailed specifications about patient's assessment tools, display and patient's transport capabilities.

\section{Discussion and conclusions}

The differences highlighted between the three health organizations point of views might be explained by the fact that NHS proposed ventilators for few hours short-term stabilisation. They also claimed that they might be used up to one day for a patient in critical condition. Ideally, these models might work as broader function ventilators for supporting patients for a limited number of days, before more advanced ventilator support becomes necessary [24]. In line with that, Alison Pittard, the dean of the Faculty of Intensive Care Medicine, confirmed that the initial request to UK industry was toward the production of simple ventilators for the early-stage treatment of COVID-19, and if patients needed a prolonged period of ventilation, a more sophisticated device would be more suitable [27, 28].

At the end of March, UK ministers ordered 10,000 extra ventilators, prioritising the production of basic devices. However, it was noted that while the overall number of new COVID-19 confirmed cases in UK was slightly decreasing, critical cases were more complicated than expected [29]. For these reasons, the UK Government decided to prioritise more sophisticated devices and cancelled an order for thousands of units of a simple ventilator model developed by industrial companies to treat COVID-19. Similar examples could be observed in Spain [30] and in France [31].

In France, for instance, the Government requested the production of 10,000 ventilators, half of which were sophisticated models, while the others had a more basic design (used in 1998). As the former are more complex and take more time to be manufactured, Government required the production of a greater number of basic design ventilators, approving the manufacture of 8500 basic and 1600 more sophisticated 
devices in 50 days. Unsurprisingly, the 8500 basic devices were considered useful for patient transport but not for treating critical patients in ICUs [32].

These examples are suggestive of a waste of time and resources, while during critical and emergency situations, costs and time are driver indicators for the manufacturing of new medical devices. However, we strongly believe that incentivizing cost-effectiveness analysis and the application of evidence-based health technology assessment methods, through the gathering, analysis and synthesis of the best available scientific evidence could have led to a reduction of unnecessary expenses, keeping the real health needs and time as driver indicators.

With these reflections, we would like to highlight how the prioritization of healthcare needs might be important. From these last examples, it clearly emerged that the frenetic race to the increase of ventilators provision was initially not counterbalanced by the quality evaluation of devices. During an emergency situation the absolute priority is saving lives and the way to better do that should be guided by scientific evidence. Structured decision-making processes based on evidence, are able to optimize time and resources allocation, evaluating the safety and effectiveness of a given medical device together with the effects of its introduction in a healthcare setting.

In this context, the comparison among different countries points of view might be crucial. We combined the answers to the COVID-19 pandemic of three Institutions that had to deal with different settings, populations, policies, etc.... However, it could be noted that the general lines issued by the three countries are similar but not identical, also because while CONSIP S.p.A and WHO extended the indications to pediatric population, the information retrieved from NHS focused only on adults. It means that Institutions should collaborate more and more in order to provide univocal reliable indications to help the world to emerge from this crisis. We strongly believe that elaborating clear indications from available scientific evidence and spreading them throughout the most renowned world Institutions, might provide significant help in this pandemic emergency, guiding and supporting the crucial decision-making processes that are affecting the life of millions people and the economic burden of all countries that are dealing with this common enemy.

Availability of data and material Not applicable.

\section{Compliance with ethical standards}

Conflicts of interest/competing interests Not applicable.

Code availability Not applicable.

Ethics approval Not applicable.
Consent to participate Not applicable.

Consent for publication Not applicable.

Conflict of interest The authors declare that they have no competing of interests.

\section{References}

1. Zhu N, Zhang D, Wang W, Li X, Yang B, Song J, et al. A novel coronavirus from patients with pneumonia in China, 2019. N Engl J Med. 2020;382(8):727-33. https://doi.org/10.1056/ NEJMoa2001017.

2. Huang C, Wang Y, Li X, Ren L, Zhao J, Hu Y, et al. Clinical features of patients infected with 2019 novel coronavirus in Wuhan. China Lancet. 2020;395(10223):497-506. https://doi.org/ 10.1016/S0140-6736(20)30183-5.

3. Wu Z, McGoogan J. Characteristics of and Important Lessons From the Coronavirus Disease 2019 (COVID-19) Outbreak in China: Summary of a Report of 72314 Cases From the Chinese Center for Disease Control and Prevention. JAMA. 2020;323(13):1 239-1242.

4. Arshad Ali S, Baloch M, Ahmed N, Arshad Ali A, Iqbal A. The outbreak of coronavirus disease 2019 (COVID-19)-an emerging global health threat. J Infect Public Health. 2020;13(4):644-6. https://doi.org/10.1016/j.jiph.2020.02.033.

5. Guan WJ, Ni ZY, Hu Y, Liang WH, Ou CQ, He JX, et al. Clinical characteristics of coronavirus disease 2019 in China. N Engl J Med. 2020;382:1708-20. https://doi.org/10.1056/NEJMoa2002032.

6. Vergano M, Bertolini G, Giannini A, Gristina G, Livigni S, Mistraletti G, et al. CLINICAL ETHICS RECOMMENDATIONS FOR THE ALLOCATION OF INTENSIVE CARE TREATMENTS, IN EXCEPTIONAL, RESOURCE-LIMITED CIRCUMSTANCES. In: SIAARTI, editor. http://www.siaarti.it/ SiteAssets/News/COVID19\%20-\%20documenti\%20SIAARTI/ SIAARTI\%20-\%20Covid-19\%20-\%20Clinical\%20Ethics\% 20Reccomendations.pdf2020.

7. Tellioğlu E, Balcı G, Mertoğlu A. Duration of stay of patients with community-acquired pneumonia in influenza season. Turk Thorac J. 2018;19(4):182-6. https://doi.org/10.5152/TurkThoracJ.2018. 17108.

8. Annuario Statistico del Servizio Sanitario Nazionale Assetto organizzativo, attività e fattori produttivi del SSN. In: Salute AEMd, editor. http://www.salute.gov.it/imgs/C_17 pubblicazioni_2879_allegato.pdf2017.

9. Perugini E. La situazione dei posti di terapia intensiva in Italia. AGI Agenzia Italia https://www.agi.it/cronaca/news/2020-03-14/ coronavirus-posti-terapia-intensiva-75308912020.

10. WHO. Health Systems Respond to COVID-19 Technical Guidance\#2 Creating surge capacity for acute and intensive care Recommendations for the WHO European Region (6 April 2020). 2020.

11. Ulteriori interventi urgenti di protezione civile in relazione all'emergenza relativa al rischio sanitario connesso all'insorgenza di patologie derivanti da agenti virali trasmissibili. Gazzetta Ufficiale 22 aprile 2020.

12. WHO. Technical specifications for invasive and non-invasive ventilators for COVID-19: Interim guidance. 2020.

13. Entire UK order of 250 Chinese ventilators ditched over danger to lives. The Guardian; 2020.

14. Baynes C. Coronavirus: hundreds of 'potentially deadly' Chinese ventilators bought by UK government ditched. Independent; 2020. 
15. Pecchia L, Pallikarakis N, Magjarevic R, Iadanza E. Health technology assessment and biomedical engineering: global trends, gaps and opportunities. Med Eng Phys. 2019;72:19-26. https://oi.org/ 10.1016/j.medengphy.2019.08.008.

16. Badnjević A, Pokvić LG, Hasičić M, Bandić L, Mašetić Z, Kovačević Ž, et al. Evidence-based clinical engineering: Machine learning algorithms for prediction of defibrillator performance 2019;54.

17. Iadanza E, Gonnelli V, Satta F, Gherardelli M. Evidence-based medical equipment management: a convenient implementation. Med Biol Eng Comput. 2019;57(10):2215-30. https://doi.org/10. 1007/s11517-019-02021-x.

18. Pecchia L, Piaggio D, Maccaro A, Formisano C, Iadanza E. The Inadequacy of Regulatory Frameworks in Time of Crisis and in Low-Resource Settings: Personal Protective Equipment and COVID-19. Health Technol (Berl). 2020:1-9. https://doi.org/10. 1007/s12553-020-00429-2.

19. WHO. Clinical management of severe acute respiratory infection (SARI) when COVID-19 disease is suspected. 2020.

20. WHO. Pocket book of hospital care for children: guidelines for the management of common childhood illnesses. . 2013.

21. WHO. Oxygen therapy for children: a manual for health workers. . 2013.

22. LINEE DI INDIRIZZO ASSISTENZIALI DEL PAZIENTE CRITICO AFFETTO DA COVID-19. Ministero della salute; 2020.
23. Rimensberger PC, Cheifetz IM, Group PALICC. Ventilatory support in children with pediatric acute respiratory distress syndrome: proceedings from the pediatric acute lung injury consensus conference. Pediatr Crit Care Med. 2015;16(5 Suppl 1):S51-60. https:// doi.org/10.1097/PCC.0000000000000433.

24. MHRA. Rapidly Manufactured Ventilator System. 2020.

25. MHRA. Rapidly Manufactured CPAP System. 2020.

26. Consip. Procedura negoziata d'urgenza Dispositivi medici per terapia intensiva e sub-intensiva destinati all'emergenza Covid-19. 2020.

27. Gallagher P. Coronavirus: UK-made short-term ventilators 'no use whatsoever' says ICU expert. iNews; 2020.

28. UK's new ventilators still awaiting regulatory green light. Financial Times; 2020.

29. Shirbon E. UK cancels order for simple ventilators, needs more complex ones: source. Reuters2020.

30. Seat detiene la producción de respiradores de emergencia por la descongestión de las UCI. Lavanguardia; 2020.

31. Monin J. Coronavirus: 8500 respirateurs produits... pour rien ? : franceinter; 2020.

32. Commande de 10000 respirateurs: mise au point du Gouvernement. Ministère des Solidarités et de la Santé; 2020.

Publisher's note Springer Nature remains neutral with regard to jurisdictional claims in published maps and institutional affiliations. 